\title{
Propeller-Pendulum for Nonlinear UAVs Control
}

\author{
http://dx.doi.org/10.3991/ijoe.v9i1.2409 \\ Tomáš Huba, Tomáš Malatinec, Mikuláš Huba \\ FEI STU in Bratislava, Bratislava, Slovakia
}

\begin{abstract}
This paper presents basic information about new experiment and about the wrapped-around learning objects for nonlinear control and other relevant topics from the mechatronics area. Its primary aim is to motivate students within the framework of the "learning by playing", "learning by discovering", or through "experiential learning” approaches to drag them to study this highly sophisticated stuff. The experiment may deal with simple but challenging positional or velocity control tasks requiring knowledge of basic physical principals of mechanics and of the associated mathematical apparatus of nonlinear differential equations. Furthermore, it is also used to master related measurement and communication problems, to carry out embedded control design and programming of embedded devices. Finally, it is also useful and illustrative in comparing traditional control methods that may be confronted towards the latest development in several areas of modern control theory.
\end{abstract}

Index Terms-nonlinear control, UAVs control, sensorics, physics, measurement, embedded control

\section{INTRODUCTION}

Challenges currently facing engineering education has been discussed already in many papers [5], [26], [32], [36]. Among many important factors one could mention demography, decreasing motivation for engineering study, decreasing preparation of young generation in math and physics, decreasing support of the society, etc. Wishing to survive, technical universities have to find ways to overcome all these issues.

Recently, development in the area of Unmanned Autonomous Vehicles (UAVs) attracted high attention both from their potential commercial users, from universities, as well as from individual enthusiasts wishing to enjoy freedom of flying [1], [7], [8], [9], [10], [13]. That all contributed to our intention to include into the preparatory course on nonlinear control also an experiment useful both for demonstrating some basic principles important for motion control as well as hardware and software important for UAVs control.

This paper discusses the main arguments behind:

- to attract students to solve problems through "learning by playing", "learning by discovering", or through "experiential learning"

- to provide them with the real-time experiments in a safe environment,

- to let them experiencing application of the theory in solving "real-life" problems,

- to show basic features of relevant control of motion from the simplest tasks to more complex ones.

\section{AIM OF THE EXPERIMENT}

UAVs control represents a complex interdisciplinary task integrating several areas as physics, mechatronics, control engineering, telecommunications, navigation, visual systems, scene recognition, etc [9] [9], [10], [13], [27], [28]. This represents a challenging task for both education and research. However, it brings also serious safety and also financial problems, since in experimenting with UAVs, each small mistake is mostly paid by damaged equipment. Therefore, it is necessary to split the overall control problem into sequence of several simpler tasks enabling to overcome the complex problems gradually, in several small steps.

To enable each student to learn by working actively and individually in a quasi-authentic framework with real time experiments, we need to have appropriately simplified plant models guaranteeing clear physical "visibility" of the controlled dynamics, i.e. a visually and auditory observable dynamics with the time constants in the range ms-few minutes, safety manipulation, reasonable price, possibly redundant availability of sensors and actuators, easy maintenance, connectivity to standard computers without special converter cards, alternatively suitable for remote experiment via internet, tasks with different level of "the control difficulty" and offering broader spectrum of dynamics to be demonstrated, etc.

By its unique features, pendulum control is broadly used to demonstrate different aspects of robust and nonlinear control [2], [3], [6], [15].

One such an experiment will focus on measurement of basic physical state variables - positional angle $\varphi$ and angular velocity $\omega=\mathrm{d} \varphi / \mathrm{dt}$ (Fig. 1), that will then be used for the positional stabilization and control.

In UAVs, basic state variables are measured by different devices as compass, gyros, accelerometers (see e.g. http://www.phidgets.com/products.php?product_id $=1056$ 0 ) that however gives signals that firstly needs to be processed to yield the required physical variables as e.g. $\varphi$ and $\omega=\mathrm{d} \varphi / \mathrm{dt}$. Students have to master this task by studying corresponding lectures of physics. To verify correctness of such a transformation, in our experiment these variables may also be directly measured by an incremental encoder (IRC) in the joint of the pendulum. Although the dynamics of flying vehicles is different from this rotating pendulum (that slightly reminds the paraglider control [24]), this experience may help students in eliminating possible errors in UAV control without the danger of crash. 
PAPER

PROPELLER-PENDULUM FOR NONLINEAR UAVS CONTROL
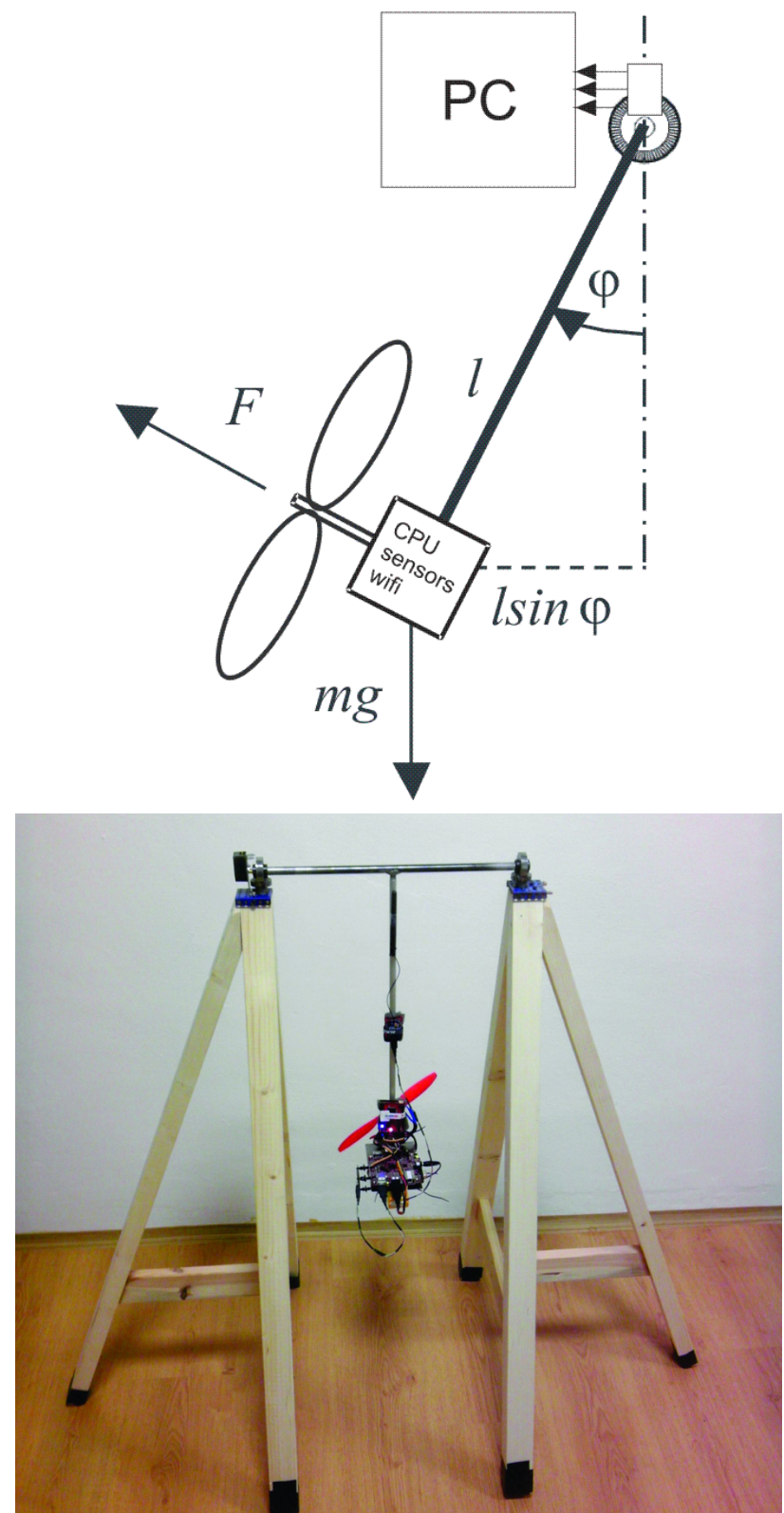

Figure 1. Simple system for constrained positional control: the schematic diagram (above) and overall appearance (below)

In order to be able to solve this task, students need also to master basics of programming languages, operating systems, electronics, communication and embedded control [10], [28].

In order to broader student's access to experimenting also out of the regular exercises, a remote access to experiment should be developed as well [16], [37].

For controlling the pendulum by manipulating voltage of the propeller motor this has to be calculated in such a way that the pendulum reaches the required position from a given initial state in a minimum time possible under different constraints on the control signal, as well as on rate of its changes. In accomplishing this task, historically, several concepts as e.g. the relay minimum time control [4], [11], [12], [19], [30], [35], robust control [2], Internal Model Control (IMC) [31], [34], Constrained PID Control [20], [21], [22], [23], flatness based intelligent PID control [14], Active Disturbance Rejection Control [17], [18], or Generalized Proportional Integral (GPI) observers [33] may be used. Students have to evaluate typical features of such approaches, their pros and cons and to propose solutions that might optimally fit potential of the much more complex problems of UAVs control.

\section{TYPICAL TASKS}

\section{A. Measurement system}

Typical tasks for the measurement system include:

- derivation and verification of transformations expressing the required signals (pendulum angle, angular velocity and acceleration) by means of trigonometric functions in terms of the measured signals from the accelerometers and gyros,

- design of optimal filters,

- reduction of gyro offset and drifts,

- analysis of the signals achieved from particular sensors,

- numerical integration of the angular velocity from the acceleration signal,

- data fusion enabling to increase measurement precision of the velocity and position signals both for rapid changes as well as smooth movements (Figure 2) and

- testing of the resulting static and dynamical precision and long-term stability of the measurement by independent measurement from incremental sensor.

Student should experience that for smoothly changing position (Figure 2, smooth) the gyro causes drifts and offset deviation from the reference values measured by the IRC. This is due to the integration of the angular velocity influenced by drifts, when the average value of the integrated signal is different from zero. For quickly changing the position value (Figure 2, fast) a centrifugal acceleration arises, which is opposite to the direction of rotation, the measured angle is much smaller than the real one shown by IRC. When the pendulum passes from smooth changes to a dynamically (rapidly) changing angle (Figure 2 , smooth to fast), it is not possible to use only one sensor, but it must be distinguished, which signal from the sensors better reproduces the true value of the position. For the fast changes it is the gyroscope and for smoother changes is it the accelerometer.

\section{B. Hardware configuration}

For real-time experiment configuration in Figure 3 has been chosen. BeagleBoard with ARM processor (1) represents one of the simplest and best ways for running algorithm on the plant in real-time and for logging data from sensors, which can be extracted from memory to desktop PC to be analyzed. This approach is appropriate both for the plant identification and reverse tuning of algorithms. For control of the BLDC motor ArduPilot(3) board is used to generate the PWM signals. All data can be extracted to PC via Xbee module. There are available two options:

- download of the saved data after ending the experiment

- receiving data during the experiment, generating the control signal and sending them back to the on-board PC. 

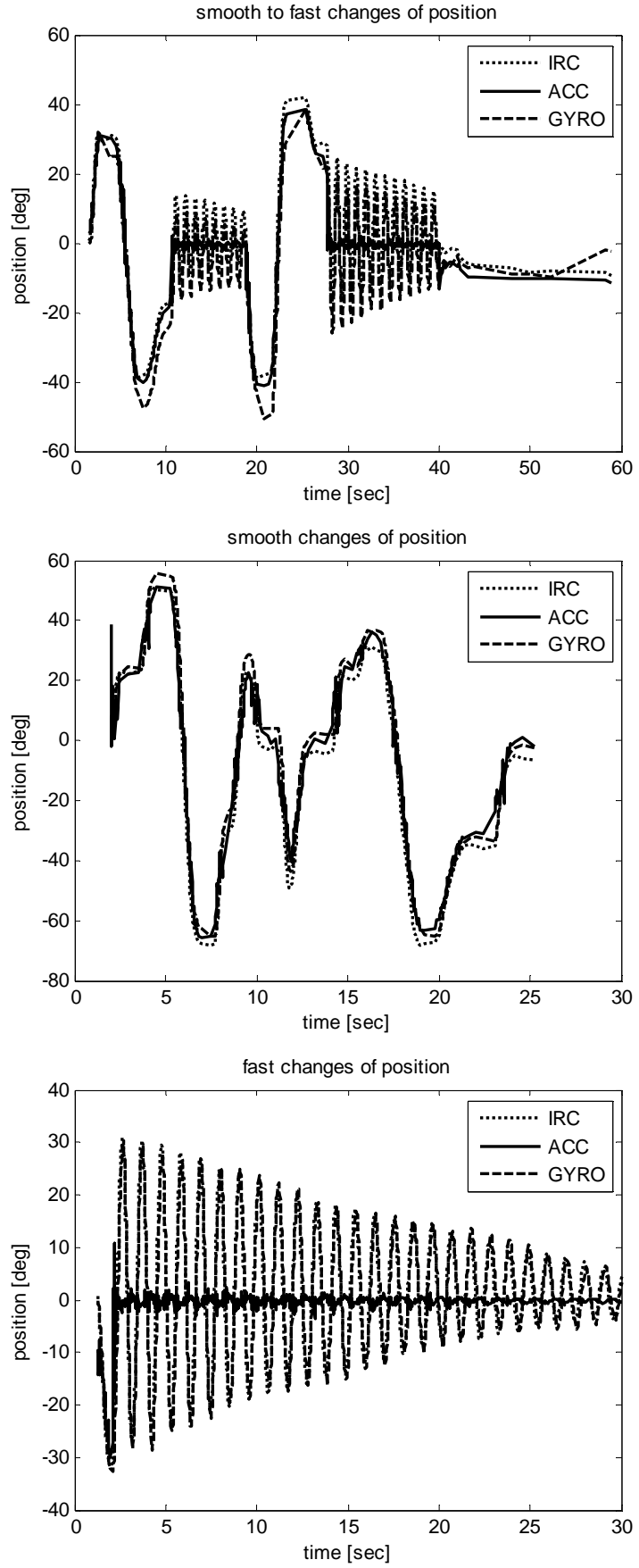

Figure 2. Comparing the position data from the accelerometers (ACC), gyro and from the incremental sensor (IRC)

IMU (inertial measurement unit) (See unit (2) in Figure $3)$ includes

- 3-axis accelerometer

- 3-axis gyroscope

- 3-axis magnetometer

and is used for logging and processing of following data

- angle from complementary filter

- angle from IRC

\section{Communication system}

In programming communication between sensors, actuators and the control unit, several typical tasks have to be mastered dealing e.g. with:

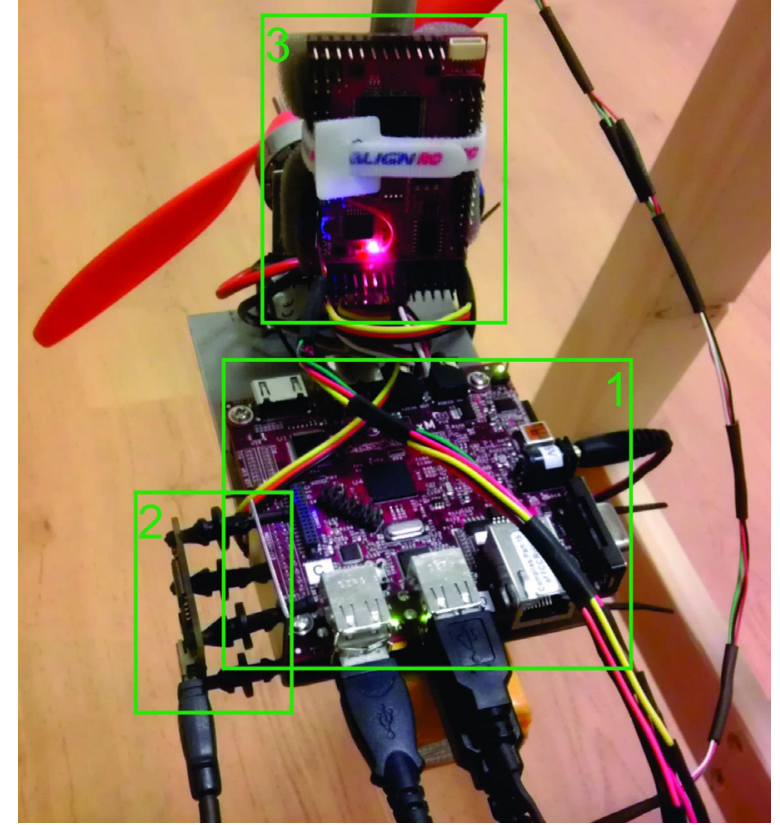

Figure 3. Detail view of electronic. 1 - BeagleBoard PC with processor unit, 2 - IMU (inertial measurement unit), 3 - ArduPilot BLDC motor control

- use of buses and protocols for communication between the device and peripherals, or between multiple devices,

- decoding and processing incoming data from a variety of peripherals and checking correctness of this information,

- testing the speed and reliability of data transmission in different communication modes.

As most computer programs that provide real time position information understand and expect data to be in NMEA National Marine Electronics Association) format [29], also the GPS receiver communication is defined within this specification. The data itself is just ASCII text and may extend over multiple sentences in certain specialized instances but is normally fully contained in one variable length sentence. Each sentence begins with a '\$' and ends with a carriage return/line feed sequence and may be no longer than 80 characters of visible text (plus the line terminators). Programs that read these data should only use the commas to determine the field boundaries and not depend on the column positions. There is a provision for a checksum at the end of each sentence which may or may not be checked by the unit that reads the data. Related tasks for students may be defined as follows:

- Connect your GPS receiver to your PC,

- Write a programme for reading data from GPS and test its correct functioning.

- Propose algorithms for parceling sentences, extract the included information and store it under variables that will be used by the control algorithm for the UAV navigation.

Peripherals used in this experiment, as e.g. the accelerometer, compass, gyro, or the barometer exist in different versions with different types of communication using different buses based on different protocols. In this experiment, the accelerometer, compass and gyro are integrated on one printed circuit and connected via USB. 
The producer delivers corresponding drivers and libraries with documentation for the most spread programming languages and operating systems. The task for students might be to write and test a programme in a chosen language for reading data from the accelerometer, gyro and compass.

The next typical problem is related to the control of the actuators (motor, servo). The available CPU does not include PWM inputs and outputs, so it is necessary to ensure the communication between CPU and actuator by means of an additional device. Possible (cheap) solution is to use a one-chip processor equipped with the PWM inputs and outputs (e.g. ATMEL/PIC). Communication between the processor and CPU is mostly using serial interface. Possible task might be to write and test a protocol for the actuator control together with the communication between CPU and actuator.

The last typical problem deals with communication between the UAV and a ground station (GS) that usually needs to be of the wireless type (RF, Wifi over GSM and $3 \mathrm{G})$. Choice of appropriate solution depends on the admissible latency, coverage by the signal, range of operation, etc. Possible task might be to design and test an appropriate wireless connection and communication protocol between the UAV and GS.

\section{Control system}

As typical control tasks related to pendulum one could mention:

- analytical and experimental identification of the pendulum dynamics,

- determination of admissible setpoint values for $\varphi$ (depending on the given torque limits defined e.g. in terms of the maximal drive voltage) and

- pendulum stabilization in a given position (with a given $\varphi$ ) - solutions to this task may depend on given torque limits, when the stabilization in upper pendulum positions may require a swing up process consisting of several control pulses with subsequently switched control signal polarity,

- reconstruction and compensation of the periodic disturbance (due to the moment of gravity) in stabilizing constant speed (angular frequency) of the pendulum rotation.

\section{PLANNING THE LEARNING ACTIVITIES}

As in each design process, planning of learning activities based on "learning by playing", "learning by discovering", or "experiential learning" has to balance a multilevel trade off between several contradictory requirements by remembering the well known facts that

- a strictly unguided instruction is extremely time consuming and can fail to meet planned instructional goals, especially without a satisfactory induction phase,

- a study group usually consists of different types of learners preferring different types of instruction and possessing different initial levels of learning and discovering skills,

- discovery learning usually does not work well for beginning and intermediary learners, so you a strongly dependant on their experience from other courses, i.e. also on the preferred teaching style of your colleagues,

- autonomous student's work may easily lead to creation of misconceptions and to pure results, especially when considering weak students.

With respect to all these potential threats, the best solutions seems to be to mix open and creative sessions giving a lot of freedom to (strong) learners (with instructor in role of observer) with guided instruction enabling to all (but especially to weak) students to benefit from a strong treatment.

E.g. in swinging up pendulum with constrained propeller torque students may discover that below some constraint torque value it is not possible to achieve the upper position by a single control pulse. You may then let the students to discuss potential solutions to the problem, to let them to verify proposed solutions, but at the end of such free session they (or you) should complete summary of potential solutions (relay minimum time control, Lyapunov-, or energy based approach, etc. - see the overview in [6]) with links to references giving the necessary details enabling to repeat the experiment with improved control algorithms.

In order to achieve the most efficient use of the time for experimenting, participation in experiments will be allowed just for sufficiently prepared students absolving successfully a pre-test. Not successful students that will not be able to find a free time to repeat the measurement within the relevant week get the chance to fill the gap at the end of semester.

Students have to upload their assignments via Moodle and to present them briefly and discuss with colleagues at the beginning of the next lesson. Pre-tests, together with post-test, presentations and discussions should help to guarantee that students not only gather the required experimenting skills, but they also absorb the required terminology and presentation skills.

\section{RELEVANT LEARNING OBJECTS}

All developed experiments will be accessible via LMS Moodle offering study materials, text of assignments, pretests, frequently asked questions, manuals and illustrative videos, upload of ready assignments, post-test, all electronic course materials containing Matlab/Simulink programmes for simulation, design and control of experiments.

\section{CONCLUSIONS}

This moment the experiment is in the development stage. It should be used from the next semester, so we are not yet able to report about its acceptance by students. After evaluating this stage of development, more complex tasks enabling to experiment in quasi-authentic environment with increased safety both for students and flying vehicles (e.g. based on differently constructed test benches) will be accomplished.

\section{ACKNOWLEDGMENT}

This work has supported by the grant VEGA-1/0656/09 Integration and development of nonlinear and robust control methods and their application in controlling flying vehicles. 


\section{REFERENCES}

[1] UAV tutorial-unmanned aerial vehicle (13.7.2012) http://www.canadafreepress.com/index.php/article/20447.

[2] Ackermann, J.: Robust Control: The Parameter Space Approach 2nd ed. Springer, Berlin, 2002. http://dx.doi.org/10.1007/978-14471-0207-6

[3] K. J. Åström and K. Furuta: swinging up a pendulum by energy control. Automatica 36 (2000) 287-295. http://dx.doi.org/ 10.1016/S0005-1098(99)00140-5

[4] Athans,M. and P.L.Falb: Optimal Control, An Introduction to the Theory and its Applications. MacGraw-Hill, New York 1966.

[5] Becker, F.S.: Why don't young people want to become engineers? Rational reasons for disappointing decisions. Europ. J. Eng. Education, 2010, 35, 4, 349-366. http://dx.doi.org/10.1080/0304 $\underline{3797.2010 .489941}$

[6] D. J. Block, K. J. Aström, and M. W. Spong, The Reaction Wheel Pendulum. Morgan \& Claypool, 2007.

[7] H. Eisenbeiss: Tutorial UAV-g Introdcution. ETH Zurich 2011. http://www.geometh.ethz.ch/uav_g/tutorial/eisenbeiss_web (13.7.2012).

[8] Unmanned Aircraft Design, Modeling and Control http://www.asl.ethz.ch/education/master/aircraft/ (13.7.2012)

[9] C. Fan; S. Guo; D. Li; Y. Liu: "Nonlinear predictive attitude control with a disturbance observer of an unmanned helicopter on the test bench," IEEE Conference on Robotics, Automation and Mechatronics (RAM), 304-309, 2011

[10] G. Farias, K.-E. Arzén, A. Cervin, S. Dormido, F. Esquembre: "Teaching Embedded Control Systems". Int. J. Engineering Education, 2010, 938-949.

[11] Feldbaum, A.A.: Optimal control systems. Academic Press, N.York, 1965.

[12] Flügge-Lotz,I.: Discontinuous and optimal control. McGraw-Hill, N.York 1968

[13] Ferruz, J.; Vega, V.M.; Ollero, A.; Blanco, V.; , "Reconfigurable Control Architecture for Distributed Systems in the HERO Autonomous Helicopter," Industrial Electronics, IEEE Transactions on , vol.58, no.12, pp.5311-5318, Dec. 2011. http://dx.doi.org/10.1109/TIE.2010.2046003

[14] Fliess, M., Join, C., 2009. Model-free control and intelligent PID controllers: 33 towards a possible trivialization of nonlinear control? In: 15th IFAC Symposium on System Identification (SYSID 2009). IFAC, Saint-Malo France.

[15] Föllinger, O.: Nichtlineare Regelungen. 7. Auflage, R. Oldenbourg Verlag München 1993.

[16] Gabriel, J.; Restivo, M.T.: "Hands-on using on-line engineering: The trend to better solutions," E-Learning in Industrial Electronics, 2009. ICELIE '09. 3rd IEEE International Conference on , 6468, 2009.

[17] Z.Gao; "Active disturbance rejection control: a paradigm shift in feedback control system design," American Control Conference, 2006.

[18] J. Han: "From PID to Active Disturbance Rejection Control," Industrial Electronics, IEEE Transactions on, vol.56, no.3, pp.900-906, 2009. http://dx.doi.org/10.1109/TIE.2008.2011621

[19] Hsu, J.C. and Mayer, A.U.: Modern Control Principles. McGraw Hill N.York 1968.

[20] Huba, M., Bisták, P.: Dynamic Classes in the PID Control. In: Proceedings of the 1999 American Control Conference. San Diego: ACC, 1999.

[21] Huba, M.: Dynamical Classes in the Minimum Time Pole Assignment Control. In: Computing Anticipatory Systems - CASYS '98. Woodbury: American Institute of Physics, 1999, 499-512. http://dx.doi.org/10.1063/1.58274
[22] Huba M., Kamenský M.: Constrained Magnetic Levitation Control. In: 16th IFAC World Congress, Prague, 2005.

[23] Huba, M., Šimunek, M.: Modular Approach to Teaching PID Control. IEEE Trans. Ind. Electr., 54, 2007, 6, 3112-3121.

[24] Huba, Tomáš - Pestún, Ivan - Huba, Mikuláš: Learning by Pleasure - Powered Paraglider and Other UAVs Control. In: ICL 2011 14th International Conference on Interactive Collaborative Learning and 11th International Conference Virtual University. Piešt'any, Slovakia.

[25] Y. Jiong; Z. Lei; D. Jiangping; S. Rong; W. Jianyu; , "GPS/SINS/BARO Integrated Navigation System for UAV," Information Technology and Applications (IFITA), 2010 International Forum on , vol.3,.19-25.

[26] Kolmos, A.: Reflections on project work and problem-based learning. Europ. J. Eng. Education, 21, 1996, 2, 141-148.

[27] Leonard, F.; Martini, A.; Abba, G.; , "Robust Nonlinear Controls of Model-Scale Helicopters Under Lateral and Vertical Wind Gusts," Control Systems Technology, IEEE Transactions on , vol.20, no.1, pp.154-163, Jan. 2012 http://dx.doi.org/10.1109/ TCST.2010.2102023

[28] P. Martí, M. Velasco, J. M. Fuertes, A. Camacho, G. Buttazzo. Design of an embedded control system laboratory experiment. "IEEE transactions on industrial electronics", 2010, vol. 57, 10, p. 3297-3307. http://dx.doi.org/10.1109/TIE.2010.2040559

[29] NMEA data http://www.gpsinformation.org/dale/nmea.htm (31.8.2012)

[30] Pontryagin L.S, Boltyanskii V.G, Gamkrelidze R. V, Mishchenko E. F, The Mathematical Theory of Optimal Processes (Russian), English translation: Interscience 1962.

[31] Rivera, D.E., Morari, M., and Skogestad, S.:. Internal model control. 4. PID controller design. Ind Eng. Chem. Res., 25,1, 1986, 252-265.

[32] Rugarcia, A., Felder, R.M., Woods, E.R., Sice, J.E.: The Future of Engineering Education: I. A Vision For a New Century. Chem. Engr. Education, 34(1), 16-25 (2000).

[33] Sira-Ramirez, H.; "On the linear control of the quad-rotor system," American Control Conference (ACC), 2011 , vol., no., pp.31783183.

[34] Skogestad, S.: Simple analytic rules for model reduction and PID controller tuning. Journal of Process Control 13, 2003, 291-309. http://dx.doi.org/10.1016/S0959-1524(02)00062-8

[35] Smith, O.J.M.: Feedback control systems. McGraw-Hill, N. York 1958.

[36] UNESCO Report Engineering: Issues, Challenges and Opportunities for Development. Unesco Publishing 2010.

[37] Žáková, K. - Kohút, M.: Matlab Based Remote Control of Thermo-Optical Plant. In: International Journal of Online Engineering. - ISSN 1861-2121. - Vol. 5, Special Issue (2009), s. 2729.

\section{AUTHORS}

Tomáš Huba, Tomáš Malatinec, and Mikuláš Huba are with the Institute of Control and Industrial Informatics, FEI STU in Bratislava, Bratislava, Slovakia (tomas.huba,tomas.malatinec,mikulas.huba@stuba.sk).

This article is an extended and modified version of a paper presented at the International Conference on Interactive Collaborative Learning (ICL2012), held 26 - 28 September 2012, in Villach, Austria. Received 30 November 2012. Published as resubmitted by the authors 24 January 2013. 